\title{
Chatting with Glue: Cognitive Tools for Augmented Conversation
}

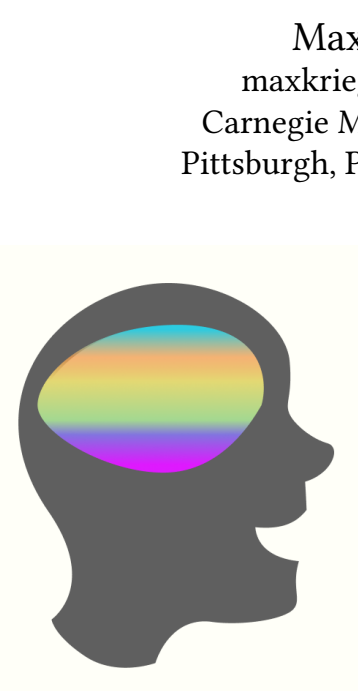

\section{ABSTRACT}

Conversation is a creative process. When we converse, we engage rich networks of meaning, forming conceptual connections within and among ourselves. To prevent these connections from fading, we take notes or work together through other external media. Sufficiently rich conversation is a multimedia activity.

The recording media we use for conversations (audio, video, text) are immutable, linear, and one-dimensional. As conversation has become computationally-mediated, it has inherited the limitations of these recording media. Both recording and chat media coerce conversation into a structure that contradicts how its content came about in the first place.

I propose tools for a more principled chat that embraces the capabilities of our minds and the computational medium. These tools augment conversation, remote or in-person, into a creative activity for knowledge-making.

\section{CCS CONCEPTS}

- Human-centered computing $\rightarrow$ Collaborative and social computing systems and tools.

\section{KEYWORDS}

conversation, chat, collaboration, media, cognitive, communication, nonlinear, comic, messaging

Permission to make digital or hard copies of part or all of this work for personal or classroom use is granted without fee provided that copies are not made or distributed for profit or commercial advantage and that copies bear this notice and the full citation on the first page. Copyrights for third-party components of this work must be honored

For all other uses, contact the owner/author(s)

$<$ Programming'20> Companion, March 23-26, 2020, Porto, Portugal

(c) 2020 Copyright held by the owner/author(s)

ACM ISBN 978-1-4503-7507-8/20/03.

https://doi.org/10.1145/3397537.3397550

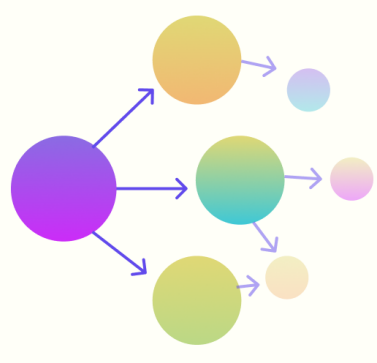

ACM Reference Format:

Max Krieger. 2020. Chatting with Glue: Cognitive Tools for Augmented Conversation. In Companion Proceedings of the 4th International Conference on the Art, Science, and Engineering of Programming (<Programming'20> Companion), March 23-26, 2020, Porto, Portugal. ACM, New York, NY, USA, 1 page. https://doi.org/10.1145/3397537.3397550

\section{THE COMIC}

This work was presented as a living, evolving comic. In it, I argue that present chat interfaces have compressed, linearized, and made immutable our conversational thinking. I then propose several affordances and principles for future chat interfaces that may resolve these contentions and ultimately augment our thinking in online conversations. These affordances, I argue, form a glue that binds thoughts together in conversation, allowing thinking to flow in a multimedia, non-linear, infinitely evolving process. Finally, I surface several future directions of inquiry for this medium.

The comic is presented in five chapters:

(1) The Paradox of Chat

(2) The Shape of Wandering

(3) Conversation Over Time

(4) The Power of Paste

(5) The Future

A snapshot of the comic, including citations and further reading, is available at doi.org/10.5281/zenodo.3889126 (DOI 10.5281/zenodo.3889126) It was created in the design application Figma.

\section{ACKNOWLEDGMENTS}

Special thanks to Cameron Burgess, Weiwei Hsu, Wode 'Nimo' Ni, Katherine Ye, Eli Parra, Gray Crawford, Jonathan Skjøtt, Jonathan Edwards, Yoshiki Schmitz, and many others. 